\title{
Investigation of the Neutrophil-to-lymphocyte Ratio and Platelet- to-lymphocyte Ratio in Patients with Neuromyelitis Optica Spectrum Disorders
}

\author{
Qingli SUN \\ Peking University Third Hospital \\ Dongsheng FAN ( $\sim$ dsfan@sina.com ) \\ Peking University Third Hospital
}

\section{Research Article}

Keywords: Neuromyelitis optica spectrum disorders, Neutrophil-to-lymphocyte ratio, Platelet-to-lymphocyte ratio

Posted Date: October 21st, 2021

DOI: https://doi.org/10.21203/rs.3.rs-955432/v1

License: (a) (1) This work is licensed under a Creative Commons Attribution 4.0 International License. Read Full License 


\section{Abstract}

Background: This study aimed to explore the differences in the neutrophil-to-lymphocyte ratio (NLR) and platelet-to-lymphocyte ratio (PLR) in patients with neuromyelitis optica spectrum disorders (NMOSDs) as well as their relationship with the onset of the diseases.

Methods: The clinical data, laboratory findings, and imaging data of patients with NMOSD admitted to Perking University Third Hospital from January 2015 to December 2020 were retrospectively analyzed. Routine blood tests of patients performed within one week of the appearance of new clinical symptoms or imaging lesions were collected to calculate the NLR and PLR. The routine blood test of the patients in remission was performed more than 6 months after the patients stopped hormone use. The NLR and PLR of patients were compared with those of 100 healthy subjects undergoing physical examinations.

Results: A total of 55 patients with NMOSD were enrolled. 44 patients with NMOSD were followed up. In patients with NMOSD, the white blood cell (WBC) count, absolute neutrophil count, and NLR were significantly higher than those in patients in remission and the controls, while the absolute lymphocyte count was significantly lower than that in patients in remission and the controls. In patients with NMOSD in remission, there were no statistically significant differences in the WBC count, absolute neutrophil count, absolute lymphocyte count, or NLR compared with the controls. The PLR of patients with NMOSD in the attack stage was significantly higher than that of the controls, while the PLR of patients with NMOSD in remission was not significantly different from that of the attack stage and the controls. There were no statistically significant differences between APQ4 (+) and APQ4 (-) in patients with NMOSD at the attack stage in the WBC count, absolute neutrophil count, absolute lymphocyte count, platelet count, NLR or PLR. ROC analysis of NLR and PLR for the diagnosis of inflammatory changes in NMOSD at the attack stage and controls: The ROC curve was plotted using NLR and PLR as dependent variables. In patients with NMOSD, the AUC was 0.806 for NLR and 0.612 for PLR. ROC analysis of NLR and PLR for the diagnosis of inflammatory changes in NMOSD at the attack stage and remission stage. The AUC was 0.728 for NLR and 0.594 for PLR.

Conclusion: Patients with NMOSD had significantly higher WBC counts, absolute neutrophil counts and NLRs, and elevated NLRs were correlated with inflammatory activity in NMOSD.

\section{Background}

Neuromyelitis optica spectrum disorders (NMOSDs) are immune-mediated demyelinating diseases of the central nervous system (CNS). They mainly affect the optic nerve and spinal cord but may also involve other parts, such as the brain, cerebellum, and brainstem, and most patients are characterized by recurrent episodes. ${ }^{[1]}$ NMOSD is likely to be combined with other autoimmune diseases, and abnormal immune responses play an important role in the development of NMOSD.

A routine blood test is one of the most commonly used blood tests in clinical practice, including indicators such as WBCs, neutrophils, and platelets. These indicators can reflect the basic inflammatory changes in the body. The neutrophil-to-lymphocyte ratio (NLR) is a biomarker for many diseases, such as myocardial infarction ${ }^{[2]}$ and chronic obstructive pulmonary disease, ${ }^{[3]}$ and an indicator for assessing prognosis. Increased neutrophil counts are correlated with inflammatory responses triggered by the relapse of MS. ${ }^{[4]}$ NLR is significantly increased in patients with MS, ${ }^{[5]}$ another demyelinating disease of the CNS and is correlated with the activity of the disease in relapsing-remitting MS. ${ }^{[6,7]}$ The platelet-to-lymphocyte ratio (PLR) is also correlated with some autoimmune diseases. ${ }^{[8,9]}$ The combination of NLR and PLR can better reflect inflammatory changes in the body. NMOSD is an autoimmune disease, and there are few studies on the correlations between NMOSD and NLR ${ }^{[10]}$ and PLR. The present study aimed to investigate the correlations among NLR, PLR, and the onset of disease in patients with NMOSD during clinical episodes.

\section{Study Subjects}

\subsection{Subjects}

The data of 55 patients with NMOSD admitted to Perking University Third Hospital from January 2015 to December 2020 were retrospectively analyzed. The inclusion criteria were as follows: (1) patients with NMOSD who met the NMOSD diagnostic criteria developed by the International NMO Diagnostic Panel in 2015. ${ }^{[1]}$ (2) patients who did not use hormones one month before the 
onset or relapse of the disease; and (3) patients with complete clinical data. The exclusion criteria were as follows: (1) patients with severe hepatic and renal dysfunction; (2) patients with malignancy, trauma, or autoimmune diseases; (3) patients with recent fever and infection; and (4) patients who were pregnant.

\subsection{Methods}

Clinical data, laboratory findings, and imaging data were collected from 55 patients with NMOSD. The clinical data included sex, age, duration of disease, number of relapses, and Expanded Disability Status Scale (EDSS) score. Laboratory tests included routine blood tests, routine urine and stool tests, hepatic and renal function tests, blood sedimentation, thyroid function and antibodies, aquaporin 4 (AQP4), tumor markers, and immune-related indicators, such as anti-nuclear antibodies (ANAs) and anti-extractable nuclear antigen antibodies (ENAs). The routine blood test of the patient performed within one week of the onset of new clinical symptoms or imaging lesions was collected as clinical data, and the NLR and PLR were calculated. Forty-four patients with NMOSD were followed up. The routine blood test of the patients in remission was performed more than 6 months after the patients stopped hormone use. The results of routine blood tests and information, such as sex and age, of 100 healthy individuals were also collected.

\subsection{Statistical analysis}

The SPSS 25.0 software package (SPSS Inc., Chicago, IL, USA) was used for data analysis. Normally distributed measurement data are expressed as the means \pm standard deviations, and nonnormally distributed measurement data are expressed as the medians (P25, P75). The t-test was used for intergroup comparisons between the normally distributed measurement data. One-way ANOVA was adopted for comparisons of the nonnormally distributed measurement data. The chi-square test or Fisher's exact probability method was used for comparisons of countable data. The diagnostic value was assessed based on the area under the curve (AUC) of the receiver operating characteristic $(\mathrm{ROC})$ curve, with $\mathrm{AUC}<0.50$ indicating no predictability, $0.50 \leq \mathrm{AUC} \leq 0.70$ indicating low predictability, $0.70<$ AUC $\leq 0.90$ indicating medium predictability, and AUC $>0.90$ indicating high predictability. $P<0.05$ was considered statistically significant.

\section{Results}

\subsection{General characteristics of patients with NMOSD:}

There were 55 patients in the NMOSD group, including 18 males and 37 females. The average age was $48.71 \pm 16.43(15-81)$ years, and 36 patients were AQP4 (+), with a positivity of $65.45 \%$. There were 36 males and 64 females in the healthy control group, with an average age of $46.45 \pm 15.71(25-80)$ years. There were no significant differences in sex or age between the three groups. The number of episodes in patients with NMOSD was $2.34 \pm 1.67$, the median duration of disease was $2(1,5)$ years, and the EDSS score was $3.70 \pm 1.58$. There were 13 males and 31 females in 44 follow-up patients, with an average age of 49.16 \pm 16.40 (21-80) years. The EDSS score was $2.28 \pm 1.34$, and 30 patients were AQP4 (+). The EDSS scores of NMOSD patients in the remission stage were significantly lower than those of NMOSD patients in the attack stage. Among the 55 patients with NMOSD, 11 took azathioprine, and 5 took mycophenolate mofelil. Among the 44 follow-up patients with NMOSD, 16 received azathioprine, and 9 received mycophenolate mofelil. There were significant differences in the use of immunosuppressants between NMOSD episodes and remission $(p=0.005)$.

\subsection{Comparison of the inflammatory indicators between patients with NMOSD at the attack stage and remission stage:}

In patients with NMOSD at the attack stage, the white blood cell (WBC) count, absolute neutrophil count, and NLR were significantly higher than those in patients in remission and the controls, while the absolute lymphocyte count was significantly lower than that in patients in remission and the controls. The differences were statistically significant. In patients with NMOSD in remission, there were no statistically significant differences in the WBC count, absolute neutrophil count, absolute lymphocyte count, or NLR 
compared with the controls. There were no statistically significant differences in the platelet count among patients with NMOSD at the attack stage, patients with NMOSD in remission, and controls. The PLR of patients with NMOSD in the attack stage was significantly higher than that of the controls, while the PLR of patients with NMOSD in remission was not significantly different from that of the attack stage and the controls. (as shown in Table 1).

Table 1

Comparison of the serological indicators among patients with NMOSD at the attack stage, in the emission stage and controls

\begin{tabular}{|lllllll|}
\hline & NMOSD $(n=55)$ & NMOSD $(n=44)$ & Control $(n=100)$ & P value* & P value $^{\#}$ & $P_{\text {value }}$ \\
\hline White blood cell $\left(x 10^{9} / \mathrm{L}\right)$ & $7.17 \pm 1.83$ & $6.16 \pm 1.28$ & $5.77 \pm 1.49$ & 0.002 & 0.000 & 0.138 \\
\hline Neutrophil $\left(x 10^{9} / \mathrm{L}\right)$ & $5.00 \pm 1.68$ & $3.71 \pm 1.14$ & $3.42 \pm 1.35$ & 0.000 & 0.000 & 0.203 \\
\hline Lymphocyte $\left(x 10^{9} / \mathrm{L}\right)$ & $1.60 \pm 0.50$ & $1.87 \pm 0.60$ & $1.88 \pm 0.48$ & 0.016 & 0.001 & 0.961 \\
\hline Platelet $\left(\mathrm{x} 10^{9} / \mathrm{L}\right)$ & $221.27 \pm 52.58$ & $223.73 \pm 42.46$ & $222.67 \pm 40.60$ & 0.802 & 0.864 & 0.887 \\
\hline NLR & $3.52 \pm 1.85$ & $2.28 \pm 1.27$ & $1.98 \pm 1.17$ & 0.000 & 0.000 & 0.158 \\
\hline PLR & $149.09 \pm 61.90$ & $129.23 \pm 40.57$ & $125.94 \pm 39.47$ & 0.058 & 0.014 & 0.648
\end{tabular}

NLR: neutrophil-to-lymphocyte ratio; PLR: platelet-to-lymphocyte ratio; P value*: comparison between NMOSD at attack stage and remission; $\mathrm{P}$ value ${ }^{\#}$ : comparison between NMOSD at attack stage and the controls; $\mathrm{P}$ value ${ }^{\Delta}$ : comparison between remission and the controls.

\subsection{Comparison of APQ4 (+) and APQ4 (-) in patients with NMOSD at the attack stage:}

there were no statistically significant differences in the WBC count, absolute neutrophil count, absolute lymphocyte count, platelet count, NLR or PLR (as shown in Table 2).

Table 2

Comparison of the general characteristics of AQP4(+) and AQP4(-) in patients with NMOSD

\begin{tabular}{|lllllllll|}
\hline & $\begin{array}{l}\text { Number } \\
\text { of } \\
\text { cases }\end{array}$ & $\begin{array}{l}\text { Sex } \\
(\text { Male/Female })\end{array}$ & $\begin{array}{l}\text { White } \\
\text { blood cell } \\
\left(\times 10^{9} / \mathrm{L}\right)\end{array}$ & $\begin{array}{l}\text { Neutrophil } \\
\left(\mathrm{x} 10^{9} / \mathrm{L}\right)\end{array}$ & $\begin{array}{l}\text { Lymphocyte } \\
\left(\mathrm{x} 10^{9} / \mathrm{L}\right)\end{array}$ & $\begin{array}{l}\text { Platelet } \\
\left(\mathrm{x} 10^{9} / \mathrm{L}\right)\end{array}$ & NLR & PLR \\
\hline $\begin{array}{l}\text { AQP4 } \\
(+)\end{array}$ & 36 & $7 / 29$ & $7.50 \pm 1.83$ & $5.24 \pm 1.67$ & $1.68 \pm 5.37$ & $218.83 \pm 48.73$ & $3.51 \pm 1.81$ & $142.94 \pm 60.25$ \\
\hline $\begin{array}{l}\text { AQP4 } \\
(-)\end{array}$ & 19 & $8 / 11$ & $6.555 \pm 1.72$ & $4.54 \pm 1.64$ & $1.45 \pm 0.40$ & $225.89 \pm 60.36$ & $3.52 \pm 1.98$ & 160.7764 .66 \\
\hline $\begin{array}{l}P \\
\text { value }\end{array}$ & - & 0.073 & 0.66 & 0.144 & 0.103 & 0.640 & 0.984 & 0.313 \\
\hline
\end{tabular}

\subsection{ROC analysis of NLR and PLR for the diagnosis of inflammatory changes in NMOSD at the attack stage and controls:}

The ROC curve was plotted using NLR and PLR as dependent variables. In patients with NMOSD, the AUC was 0.806 for NLR ( $P=$ $0.000,95 \% \mathrm{Cl}: 0.735$ to 0.876$)$ and 0.612 for PLR ( $=0.022,95 \% \mathrm{Cl}: 0.519$ to 0.705$)$. The optimal cutoff point was 0.727 , the sensitivity was $72.7 \%$, and the specificity was $75 \%$, as shown in Figure 1. ROC analysis of NLR and PLR for the diagnosis of inflammatory changes in NMOSD at attack stage and remission stage邓the AUC was 0.728 for NLR ( $P=0.000,95 \%$ Cl: 0.629 to 
0.828 ) and 0.594 for PLR ( $P=0.108,95 \% \mathrm{Cl}: 0.482$ to 0.706$)$. The optimal cutoff point was 2.3045 , the sensitivity was $72.7 \%$, and the specificity was $63.6 \%$, as shown in Figure 2.

\section{Discussion}

Neutrophils are the most abundant cell type in the innate immune system and are associated with the pathogenesis of NMOSD. ${ }^{[11,12]}$ Studies on animal models of experimental autoimmune encephalomyelitis have shown that the reduction in neutrophils may delay disease progression. Neutrophils may play an important role in the pathogenesis of experimental autoimmune encephalomyelitis by producing cytokines, damaging the blood-brain barrier, and promoting inflammation in the brain parenchyma. ${ }^{[13]}$ It is now well established that neutrophils are the main inflammatory cells involved in early pathogenesis in models of NMOSD. ${ }^{[14]}$ Inflammatory cells infiltrating the lesions in NMOSD mainly include neutrophils, macrophages, and eosinophils, with relatively few T lymphocytes. ${ }^{[11]}$ During the pathogenesis of NMOSD, neutrophils are the first inflammatory cells to enter the lesion; entering may occur within a few hours after the onset of inflammation. Within 12 hours after onset, perivascular complement activation and loss of AQP4 with early myelin loss occur. In the chronic phase (seven days), there were almost no

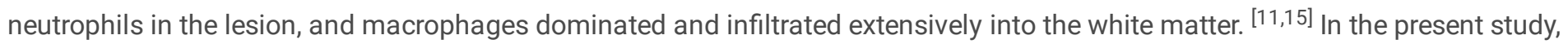
the WBC counts and absolute neutrophil counts during episodes of NMOSD were significantly higher than those in remission of NMOSD and the control group, whereas in patients with NMOSD in remission, there were no statistically significant differences compared with the controls, suggesting that neutrophils play a role during episodes of NMOSD. This finding is consistent with the pathophysiological process of NMOSD. Therefore, treatment for NMOSD should block the persistent inflammation produced by the apoptosis of neutrophils, promote the apoptosis of neutrophils, enhance the regression of inflammation, inhibit excessive tissue injury, and avoid persistent chronic inflammation. ${ }^{[16]}$

The NLR is a systemic inflammatory marker based on routine blood tests, which are low in cost and easy to obtain. The NLR can reflect both the increase in neutrophils and the decrease in lymphocytes during the inflammatory response. The NLR has been correlated not only with some autoimmune diseases ${ }^{[9,17]}$ but also with neurological disorders, such as Parkinson's disease ${ }^{[18]}$ and Alzheimer's disease. ${ }^{[19]}$ Several studies have verified that the NLR is correlated with the prognosis of diseases such as rheumatoid arthritis, ${ }^{[9]}$ cancer, ${ }^{[20,21]}$ and stroke. ${ }^{[22]}$

Studies concerning NLR and NMOSD are rarely reported. Neutrophils are involved in the pathogenesis of NMOSD, ${ }^{[11,13,14]}$ and the NLR can reflect changes in neutrophils in vivo. The present study revealed that the NLR was significantly higher in patients experiencing episodes of NMOSD than in patients in remission and controls. The change in NLR was due to an increase in the absolute neutrophil count and a decrease in the absolute lymphocyte count. Thus, it was suggested that the NLR could be used as one of the peripheral inflammatory biomarkers of disease activity in patients with NMOSD. The NLR and absolute neutrophil counts of patients with NMOSD at the attack stage were significantly higher than those of patients in remission, indicating that the inflammatory response in NMOSD onset might be more intense than that in remission, which also verified the difference in pathogenesis between the two different stages. This result suggests that the index has a moderate prediction value, indicating that the NLR might be a useful additional peripheral inflammatory biomarker in assessing disease activity in patients with NMOSD.

PLR is gaining importance as an indicator of inflammation. PLR in combination with NLR can better reflect the changes in inflammation in vivo and avoid the influence of other factors on the absolute counts of leukocyte subtypes. The present study revealed no statistically significant differences in the absolute platelet count between patients with NMOSD at the attack stage, remission stage and the controls, but the absolute lymphocyte count of patients experiencing episodes of NMOSD was significantly lower than that of patients in remission and the controls. The PLR of patients with NMOSD attack stage was significantly higher than that of the controls, which may make the decrease of absolute lymphocytes count of patients with NMOSD attack stage larger. There was a significant decrease in absolute lymphocyte count during episodes in patients with NMOSD, but there was no consistent change in PLR. There was no significant difference in PLR between the NMOSD attack stage and remission stage, but $P$ $=0.058$, which was close to statistical significance. This value might be correlated with the small number of patients with NMOSD, so it would be necessary to expand the number of cases in further studies. This study investigated the sensitivity and specificity of NLR and PLR to inflammatory changes in NMOSD patients by drawing ROC curves. It was found that the changes in NLR in 
patients with attack stage were statistically significant compared with those in remission stage and the controls and better reflected inflammatory changes than PLR.

Drug use in patients with NMOSD may affect the WBC count, absolute neutrophil count, and absolute lymphocyte count, thereby affecting NLR and PLR. Some NMOSD patients took immunosuppressants in the onset and remission stages, and the proportion of immunosuppressants in the remission stage was significantly higher than that in the onset stage, which may partially affect NLR and PLR.

In conclusion, NLR might significantly increase in patients with NMOSD during episodes, and the increase in NLR might be correlated with inflammatory activity in NMOSD. The NLR could be used as a peripheral inflammatory biomarker of disease activity during an episode in patients with NMOSD and has some clinical applications. NLR can be a useful complement to MRI and CSF in the diagnosis of MS disease and is easier to achieve than the latter. However, the present study was a single-center study, and the number of patients enrolled was relatively small. The patients were taking different medications before relapse and after relapse, which might affect the results and have certain limitations. Further expansion is needed to increase the number of patients.

\section{Declarations}

\section{Ethics approval and consent to participate}

This study was reviewed and approved by the Peking University Third Hospital Medical Science Research Ethics Committee (Item NO. 2021190). And this Ethics Committee exempted the informed consent due to retrospective nature of this study. All methods were carried out in accordance with the Declaration of Helsinki.

\section{Consent for publication}

Not applicable.

\section{Availability of data and materials}

All data and material supporting the conclusions of this article is included in the article.

\section{Competing interests}

The authors declare that they have no competing interests.

\section{Funding}

None.

\section{Authors' contributions}

DSF contributed to the concept and design of the manuscript. QLS performed literature review for the manuscript and wrote the manuscript. All authors contributed to revising the manuscript, reading, and approving the submitted version

\section{Acknowledgements}

We thank Aping Sun, Yan Ma for cases collected.

\section{References}


1. Wingerchuk DM, Brenda B, Bennelt JL. International consensus diagnostic criteria for neuromyelitis optica spectrum disorders [J].Neurology, 2015,85(2):177-189.

2. Nunez J, Nunez E, Bodi V, et al. Usefulness of the neutrophil to lymphocyte ratio in predicting long-term mortality in ST segment elevation myocardial infarction [J]. Am J Cardiol, 2008, 101(6):747-752.

3. Gunay E, Sarinc Ulasli S, Akar O, et al. Neutrophil to-lymphocyte ratio in chronic obstructive pulmonary disease: A retrospective study [J]. Inflammation, 2014,37(2):374-380.

4. Tillack K, Naegele M, Haueis $C$, et al. Gender differences in circulating levels of neutrophil extracellular traps in serum of multiple sclerosis patients[J]. J Neuroimmunol, 2013,261(1-2):108-119.

5. Demirci S, Demirci S, Kutluhan S, et al. The clinical significance of the neutrophil-to-lymphocyte ratio in multiple sclerosis [J]. Int J Neurosci, 2016,126(8):700-706.

6. D'Amico E, Zanghì A, Romano A, et al. The neutrophil-to-lymphocyte ratio is related to disease activity in relapsing remitting multiple sclerosis [J]. Cells, 2019,8(20):1114.

7. Bisgaard AK, Pihl-Jensen G, Frederiksen JL. The neutrophil-to-lymphocyte ratio as disease actvity marker in multiple sclerosis and optic neuritis [J]. Mult Scler Relat Disord, 2017,18: 213-217.

8. Qin B, Ma N, Tang Q, et al. Neutrophil to lymphocyte ratio (NLR) and platelet to lymphocyte ratio (PLR) were useful markers in assessment of inflammatory response and disease activity in SLE patients [J]. Mod Rheumatol, 2016,26(3):372-376.

9. Fu H, Qin B, Hu Z, et al. Neutrophil-and platelet-to-lymphocyte ratios are correlated with disease activity in rheumatoid arthritis [J]. Clin Lab, 2015,61(3-4):269-273.

10. Lin J, Xue B, Li J, Xu,et al. Neutrophil to lymphocyte ratio may be a helpful marker to evaluate disease activity in NMOSD [J]. Neurol Sci, 2017,38:1859-1863.

11. Saadoun S, Waters P, MacDonald C, et al. Neutrophil protease inhibition reduces neuromyelitis optica-immunoglobulin Ginduced damage in mouse brain [J]. Ann Neurol, 2012,71: 323-333.

12. Zhang $\mathrm{H}$, Verkman AS. Eosinophil pathogenicity mechanisms and therapeutics in neuromyelitis optica[J]. J Clin Invest, 2013,123: 2306-2316.

13. Pierson ER, Wagner CA and Goverman JM. The contribution of neutrophils to CNS autoimmunity [J]. Clin Immunol, 2018,189: 23-28.

14. Ye Gong, Ya-ling Zhang, Zhen Wang,et al. Tanshinone IIA alleviates brain damage in a mouse model of neuromyelitis optica spectrum disorder by inducing neutrophil apoptosis[J]. J Neuroinflammation, 2020,17(1):198.

15. Saadoun S, Waters P, Macdonald C, et al. T cell deficiency does not reduce lesions in mice produced by intracerebral injection of NMO-IgG and complement[J]. J Neuroimmunol, 2011, 235:27-32.

16. Gilroy DW, Lawrence T, Perretti M, Rossi AG. Inflammatory resolution: new opportunities for drug discovery [J]. Nat Rev Drug Discov. 2004,3: 401-416.

17. Acarturk G, Acay A, Demir K, et al. Neutrophil-to-lymphocyte ratio in inflammatory bowel disease-as a new predictor of disease severity[J]. Bratisl Lek Listy, 2015,16: 213-217.

18. Akil E, Bulut A, Kaplan I, et al. The increase of carcinoembryonic antigen (CEA), high-sensitivity C-reactive protein, and neutrophil/lymphocyte ratio in Parkinson's disease [J]. Neurol Sci, 2015,36: 423-428.

19. Kuyumcu ME, Yesil Y, Ozturk ZA, et al. The evaluation of neutrophil-lymphocyte ratio in Alzheimer's disease [J]. Dement Geriatr Cogn Disord, 2012,34: 69-74.

20. Yugui Sun, Lifei Zhang. The clinical use of pretreatment NLR, PLR, and LMR in patients with esophageal squamous cell carcinoma: evidence from a meta-analysis [J]. Cancer Manag Res, 2018, 22(10):6167-6179.

21. Wei $B$, Yao M, Xing C, et al. The neutrophil lymphocyte ratio is associated with breast cancer prognosis: An updated systematic review and meta-analysis [J]. OncoTargets Ther, 2016,9: 5567-5575.

22. Tokgoz S, Kayrak M, Akpinar Z, et al. Neutrophil lymphocyte ratio as a predictor of stroke[J]. J Stroke Cerebrovasc Dis, 2013,22:1169-1174.

\section{Figures}




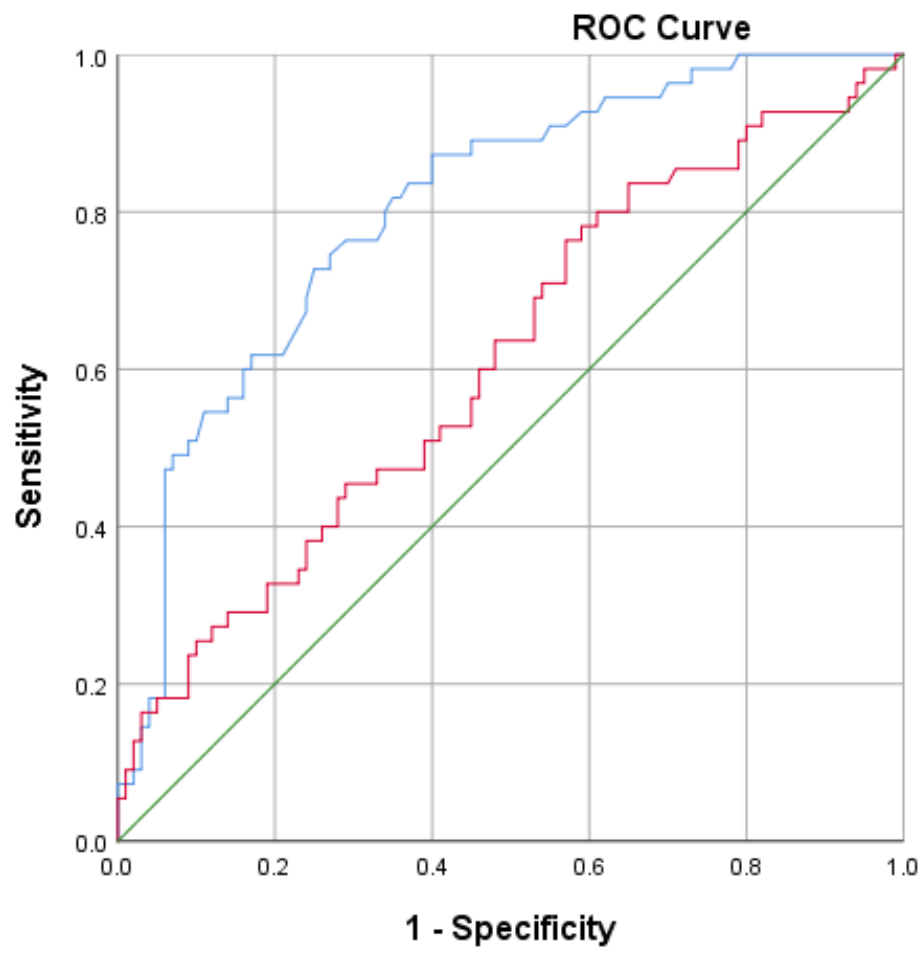

\section{Source of the Curve \\ - NLR \\ - PLR \\ - Reference Line}

Diagonal segments are produced by ties.

\section{Figure 1}

Receiver operating characteristic (ROC) curves of NLR and PLR for the diagnosis of inflammatory changes in patients with NMOSD during relapse compared to healthy controls

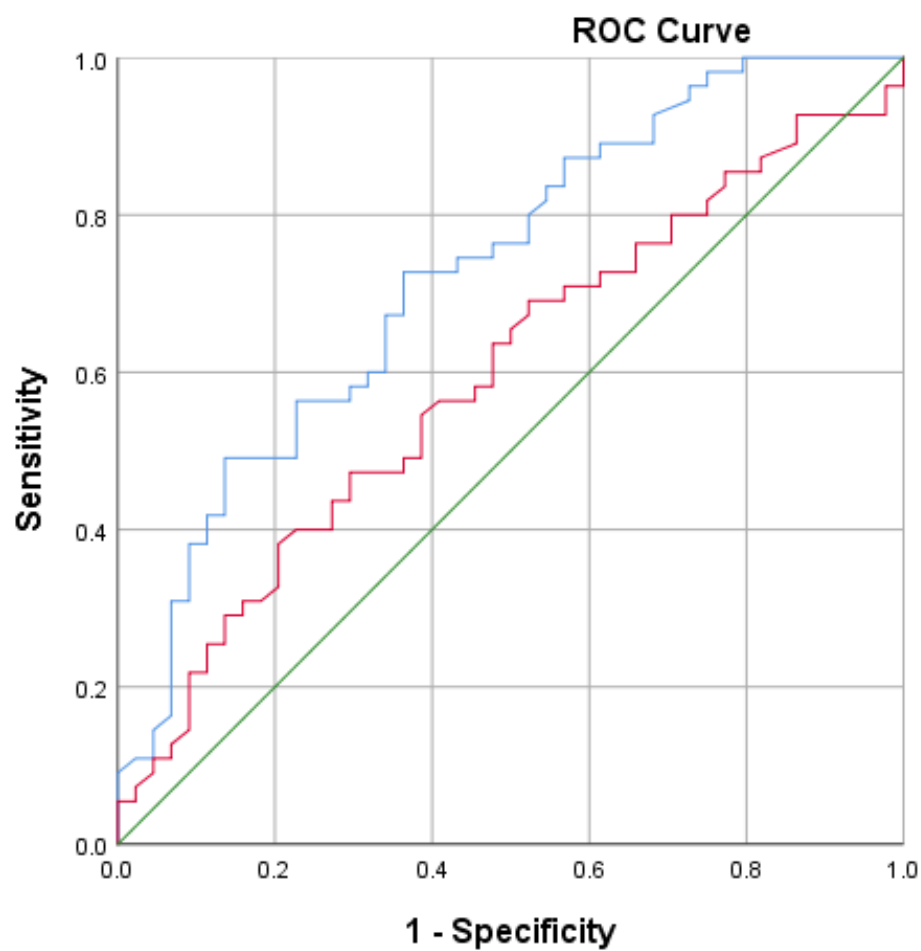

\section{Source of the} Curve

NLR

-PLR

- Reference Line

Diagonal segments are produced by ties.

Figure 2 
The receiver operating characteristic (ROC) curves of NLR and PLR for the diagnosis of inflammatory changes in patients with NMOSD during relapse compared to those in the remission stage. 